\title{
O USO DE SIMULADOR NO ENSINO DE AVALIAÇÃO CLÍNICA EM ENFERMAGEM ${ }^{1}$
}

\author{
Carla Regina de Souza Teixeira², Luciana Kusumota, Fernanda Titareli Merizio Martins Braga4, Vanessa \\ Pirani Gaioso ${ }^{5}$, Cláudia Benedita dos Santos 6 , Vivian Libório de Sousa e Silva ${ }^{7}$ Emilia Campos de Carvalho ${ }^{8}$
}

\footnotetext{
${ }^{1}$ Pesquisa financiada pelo Edital MCT/CNPq n ${ }^{\circ}$ 014/2008 - Universal. Projeto de Pesquisa “Enfermagem: competências e evidências". Processo 473758/2008-3.

2 Doutora em Enfermagem. Professora Doutora do Departamento de Enfermagem Geral e Especializada (DEGE) da Escola de Enfermagem de Ribeirão Preto (EERP) da Universidade de São Paulo (USP). São Paulo, Brasil. E-mail: carlarst@eerp.usp.br

${ }^{3}$ Doutora em Enfermagem. Professora Doutora do DEGE/EERP/USP. São Paulo, Brasil. E-mail: kusumota@eerp.usp.br

${ }^{4}$ Doutoranda em Enfermagem do Programa de Enfermagem Fundamental da EERP/USP. Especialista de Laboratório do DEGE/EERP/USP. São Paulo, Brasil. E-mail: titareli@eerp.usp.br

${ }^{5}$ Doutoranda do Department of Family/Child Health and Caregiving. University of Alabama at Birmingham. School of Nursing Birmingham, United States of America. E-mail: vgaioso@uab.edu

${ }^{6}$ Doutora em Matemática. Professora Associado do Departamento de Enfermagem Materno e Infantil da EERP/USP. São Paulo, Brasil. E-mail: cbsantos@eerp.usp.br

${ }^{7}$ Aluna do $6^{\circ}$ semestre do Curso de Graduação em Enfermagem-Licenciatura da EERP/USP. Especialista de Laboratório do DEGE/EERP/USP. São Paulo, Brasil. E-mail: liborio_vi@hotmail.com

${ }^{8}$ Doutora em Enfermagem. Professor Titular do DEGE/EERP/USP. São Paulo, Brasil. E-mail: ecdcava@usp.br
}

RESUMO: Objetivo: avaliar o uso de simulador no ensino da avaliação clínica dos olhos. Material e Método: estudo experimental randomizado, controlado com 31 alunos do $2^{\circ}$ ano de Graduação em Enfermagem de uma escola do interior paulista. A coleta de dados ocorreu de setembro a outubro de 2009, com dois instrumentos previamente validados, após autorização do Comitê de Ética. Os sujeitos foram divididos em Grupo 1 (ensino tradicional e o uso do simulador de baixa fidelidade) e Grupo 2 (ensino tradicional) para a avaliação dos olhos. Resultados: houve aumento de acertos pelos sujeitos nas questões teóricas após a aula expositiva dialogada. Na avaliação prática clínica dos olhos a média percentual de acertos foi de $76 \pm 7$ (Grupo 1) e 21 \pm 11 (Grupo 2) (p < 0,01). Conclusão: O uso do simulador mostrou ser estratégia facilitadora no processo ensino-aprendizagem.

DESCRITORES: Ensino. Materiais de ensino. Enfermagem.

\section{USE OF SIMULATOR IN TEACHING NURSING CLINICAL EVALUATION}

\begin{abstract}
Objective: to evaluate the use of simulators in teaching clinical eye examinations/evaluations. Material and Methods: experimental, randomized, and controlled study, involving 31 second-year undergraduate nursing students from a school in the interior São Paulo, Brazil. Data was collected between September and October of 2009, using previously validated instruments and after authorization from the University Ethics Committee. Subjects were divided into Group 1 (traditional teaching and use of lowfidelity simulator), and Group 2 (traditional teaching) for evaluation of the eyes. Results: the number of right answers by subjects in theoretical questions increased after a dialogued, expositive class. In the practical eye clinical evaluation, the average percentage of right answers was $76 \pm 7$ (Group 1) and $21 \pm 11$ (Group 2) $(p<0,01)$. Conclusion: The use of simulator was shown to be a facilitating strategy in the teaching-learning process
\end{abstract}

DESCRIPTORS: Teaching. Teaching materials. Nursing.

\section{EL USO DE SIMULADOR EN LA ENSEÑANZA DE EVALUACIÓN CLÍNICA EN ENFERMERÍA}

\begin{abstract}
RESUMEN: Objetivo: evaluar el uso de simulador en la enseñanza de evaluación clínica de los ojos. Material y Método: estudio experimental, aleatorizado y controlado, con 31 alumnos del segundo año de Pregrado en Enfermería de una escuela del interior del estado de São Paulo. La recolección de datos ocurrió entre septiembre y octubre de 2009, con dos instrumentos previamente validados y con la autorización del Comité de Ética. Para la evaluación de los ojos, los sujetos fueron divididos en: Grupo 1 (enseñanza tradicional y uso del simulador de baja fidelidad), y Grupo 2 (enseñanza tradicional) Resultados: hubo aumento de aciertos por los sujetos en las cuestiones teóricas después de la clase expositiva dialogada. En la evaluación práctica clínica de los ojos, el promedio porcentual de aciertos fue de $76 \pm 7$ (Grupo 1) y 21 \pm 11 (Grupo 2) (p<0,01). Conclusión: El uso del simulador mostró ser estrategia facilitadora en el proceso de enseñanza-aprendizaje.
\end{abstract}

DESCRIPTORES: Enseñaza. Materiales de enseñaza. Enfermería. 


\section{INTRODUÇÃO}

O ensino de Enfermagem tem sofrido várias transformações, com o passar dos anos para atender as necessidades de uma sociedade, também em contínua mudança e evolução. Nessa perspectiva, tem-se investigado o emprego de novas tecnologias, como uma estratégia de ensino-aprendizagem, na qual se busca o desenvolvimento e aprimoramento de habilidades necessárias para as atividades assistenciais futuras. ${ }^{1}$

Dentre as tecnologias existentes, a simulação clínica com o emprego dos simuladores é destacada na literatura como uma estratégia que possibilita o desenvolvimento do pensamento crítico, bem como o aumento da habilidade para a avaliação e decisão clínica que será requerida na prática assistencial. ${ }^{2-4}$

A simulação clínica propõe-se a replicar aspectos reais e essenciais da clínica em cenário de aprendizado, com a conveniência de simular diversas situações possíveis, o que enriquece o processo ensino-aprendizagem. ${ }^{5}$

O uso de simuladores tem garantido um ambiente seguro para a realização das intervenções de enfermagem, o que contribui para o fortalecimento, no aluno, da sua autoconfiança. Os estudantes têm a possibilidade de cometer erros, corrigi-los e aprender com eles, sem provocar dano ao paciente. Estudantes relataram alta satisfação no ensino de habilidades quando a estratégia de simulação foi empregada. Referiram sentir uma maior motivação em realizar as intervenções, sem medo de tentar e fazer perguntas, ${ }^{4}$ contribuindo, assim, com a melhoria das reações psicológicas que afloram, por meio de sentimentos de insegurança, medo e incerteza que os alunos sentem durante a realização das intervenções de enfermagem. ${ }^{6}$

Estudos prévios recomendam o uso de simuladores no ensino de semiologia para os cursos de enfermagem, pois estes recursos possibilitam diferenciar achados normais e anormais, bem como identificar as respostas fisiológicas. ${ }^{4}$ Nenhum estudo foi encontrado sobre o uso de simuladores no ensino de enfermagem na literatura cientifica nacional. Encontrou-se apenas alguns estudos na área médica, especificamente com médicos residentes em anestesiologia. ${ }^{7-8} \mathrm{No}$ ensino de enfermagem, quatro estudos realizaram simulação assistida por computador e o uso de software. ${ }^{9-12}$ Contudo, um destes estudos ressaltou a dificuldade na transferência de conhecimento para a vida real dos estudantes, pois o contato do aluno fica apenas no mouse e no teclado ${ }^{10}$ e não na prática mais realística, como seria com o uso de simuladores.

Os simuladores podem ser classificados como de baixa, moderada ou de alta fidelidade, de acordo com sua capacidade de reproduzir precisamente sons ou imagens. Os simuladores de baixa fidelidade são caracterizados como os simuladores estáticos, menos realísticos e usados para procedimentos específicos, como exemplo, os manequins de espuma usados para a prática de injeção intramuscular. Os simuladores de moderada fidelidade são mais realísticos, pois podem oferecer ausculta de sons respiratórios, cardíacos, pulsação, ou a identificação de diferentes diagnósticos. Os simuladores de alta fidelidade são manequins extremamente realísticos, pois muitos possuem movimentação torácica, olhos funcionais que piscam e reagem com a luz, sons cardíacos, pulmonares, gastrointestinais e vocais, apresentam sangramentos e secreções e reagem de acordo com as intervenções realizados pelos estudantes. ${ }^{13}$

Uma das limitações na disseminação do uso dos simuladores de alta fidelidade é o alto custo, variando entre 28 mil e 150 mil dólares, sendo que até o momento nenhuma empresa brasileira fornece estes produtos.

Assim, nesta pesquisa, optou-se por usar um simulador de baixa fidelidade, que é um modelo ou manequim usado para a prática de manobras ou procedimentos mais simples, como o simulador para treinamento de retinopatia. Ele permite praticar o procedimento de exame dos olhos por meio da inspeção direta ou com o auxílio de um oftalmoscópio, com ênfase no achado clínico de retinopatia diabética. No cuidado de enfermagem, a avaliação visual sistemática, incluindo o uso do oftalmoscópio, tem sido uma prática ainda em construção, tanto na docência como na assistência. ${ }^{14}$ Então, optou-se por realizar uma avaliação do desempenho de alunos do $2^{\circ}$ ano do Curso de Graduação em Enfermagem (Bacharelado), na avaliação clínica dos olhos, segundo a utilização ou não do simulador, associado ao método tradicional no ensino.

\section{METODOLOGIA}

Trata-se de um estudo de natureza experimental, controlado, randomizado, realizado com 31 alunos regularmente matriculados no $2^{\circ}$ ano do Curso de Graduação em Enfermagem (Bacharelado) de uma escola do interior paulista. 
Neste período se inicia formalmente o ensino de técnicas e métodos do exame clínico de adulto para obtenção de dados objetivos e subjetivos, referentes aos diferentes sistemas corporais, incluindo a avaliação clínica dos olhos.

Para este estudo, os alunos foram divididos em dois grupos, de forma aleatória, sendo que o Grupo 1 teve o ensino de forma tradicional, com aula expositiva dialogada, demonstração pelo professor e a prática em laboratório com o simulador de exame dos olhos. O Grupo 2 teve o ensino de forma tradicional com aula expositiva dialogada e demonstração pelo professor em laboratório sem o uso do simulador.

Os seguintes critérios de inclusão foram empregados para a realização deste estudo: ser aluno regularmente matriculado no $4^{\circ}$ semestre do Curso de Graduação em Enfermagem (Bacharelado) e aceitar em participar do estudo, por meio da assinatura do termo de consentimento da pesquisa. Foram excluídos quatro sujeitos que não cumpriram qualquer uma das fases do estudo, ou que manifestaram desejo de interromper sua participação.

O estudo foi desenvolvido em quatro etapas: elaboração e validação do instrumento de coleta de dados; pré-teste do instrumento de coleta de dados; atividade de ensino-aprendizagem; coleta e análise dos dados. Foram elaborados dois instrumentos de coleta de dados para identificação do conhecimento básico na avaliação clínica dos olhos. O primeiro continha 32 questões teóricas que possibilitaram identificar o conhecimento do aluno na avaliação clínica dos olhos. As questões apresentavam alternativas de verdadeiro ou falso para que o aluno assinalasse a sua opinião. $\mathrm{O}$ segundo instrumento de coleta de dados foi elaborado na forma de lista de itens para a análise dos passos do procedimento de avaliação clínica dos olhos, no total de 55 itens. Os dois instrumentos de coleta de dados foram submetidos à avaliação de três juízes, enfermeiros, docentes que ministram disciplina de Semiologia e Semiotécnica em Enfermagem, na qual foram avaliados o conteúdo e a aparência, a clareza dos dados, a facilidade da leitura, o conteúdo e apresentação do instrumento, além de sugestões acerca da necessidade de retirada, acréscimo ou alterações dos dados estabelecidos previamente. ${ }^{15}$

Foi realizado um pré-teste com o instrumento de coleta de dados por três alunos de graduação em enfermagem, que não faziam parte da amostra, para avaliar a aplicabilidade do instrumento e pro- mover as modificações necessárias. Optou-se por incluir a alternativa "não sabe" no instrumento de avaliação cognitiva, e na avaliação prática incluiu-se espaço para o registro do avaliador quanto aos acertos e erros referentes às estruturas anatômicas externas dos olhos.

A coleta de dados foi realizada por dois enfermeiros devidamente treinados pelos pesquisadores. Um profissional independente dessa pesquisa (aluno de pós-graduação em enfermagem), supervisionado pelo pesquisador principal, identificou os sujeitos da pesquisa que atenderam aos critérios de inclusão adotados para o presente estudo e explicou os objetivos da pesquisa, esclarecendo o tipo de participação dos alunos na pesquisa. Foi destinada uma semana para reflexão sobre o desejo ou não de ingressar. Após o consentimento do sujeito em participar da pesquisa, foi solicitado que assinasse o termo de consentimento do estudo. Dessa forma, 36 alunos concordaram em participar do estudo.

Inicialmente, foi aplicado o primeiro instrumento de coleta de dados para identificação do conhecimento prévio dos alunos na avaliação clínica dos olhos. Em seguida, no primeiro momento, os alunos foram submetidos ao ensino de forma tradicional com aula expositiva dialogada, tendo duração de duas horas. Ao término, foi aplicado o mesmo instrumento, sendo que quatro alunos não completaram essa fase da pesquisa, e um aluno faltou nesse dia, totalizando então 31 alunos que completaram a primeira fase do estudo.

No segundo momento, os 31 alunos foram divididos em dois grupos, de forma aleatória, sendo que o Grupo 1 teve o ensino de forma tradicional com aula expositivo-dialogada, demonstração pelo professor e a prática em laboratório com o simulador de olho (grupo de intervenção). O Grupo 2 teve o ensino de forma tradicional com aula expositivo-dialogada e demonstração pelo professor, em laboratório, sem o uso do simulador (grupo controle). Ao término, foi aplicado o segundo instrumento de coleta de dados para a avaliação prática individual, com duração média de sete minutos. A coleta de dados ocorreu de setembro de 2009 a outubro de 2009, em horário diferente das atividades curriculares. Após a conclusão da pesquisa foi realizado o ensino com o uso de simuladores para os alunos do grupo controle.

Os dados coletados foram, inicialmente, organizados e digitados em planilha eletrônica única no Microsoft Excel. Posteriormente, foram analisados e processados no programa estatístico Statical Packa- 
ge for Social Sciences (SPSS) for Windows, módulos base, e exact test versão 14.0. Para a análise estatística utilizou-se do teste de Kolmogorov-Smirnov para avaliar a distribuição da normalidade e após o teste t de Student. O nível de significância estatística adotado foi de $5 \%(\alpha=0,05)$.

O projeto de pesquisa foi encaminhado ao Comitê de Ética em Pesquisa da instituição de ensino que sediou o estudo, sendo aprovado (Protocolo $n^{\circ} 1024$ / 2009).

\section{RESULTADOS}

Os resultados obtidos no teste de Kolmogorov-Smirnov para as variáveis em estudo confirmaram a distribuição normal dos dados. A média percentual de acertos dos sujeitos do estudo $(n=31)$, relacionados às questões teóricas aplicadas antes e após a aula expositivo-dialogada, e referente ao desempenho na avaliação prática dos olhos, evidenciaram mudanças entre os grupos e os momentos de avaliação (Tabela 1 e 2).

O Grupo $1(n=15)$ apresentou média percentual de $32 \pm 13$ acertos antes da aula expositivo-dia-

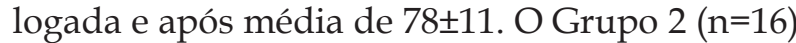
apresentou média percentual de 39 \pm 12 antes da aula expositivo-dialogada, e após, a média de $82 \pm 7$. Pode-se observar que tanto o Grupo 1 , como o Grupo 2, apresentaram desempenho menor no pré-teste realizado antes da aula expositivo-dialogada, quando comparados aos resultados obtidos após a aula. Ressalta-se que não houve diferença com significância estatística para as mudanças nos escores médios obtidos pelos dois grupos, em ambos os momentos, antes $(\mathrm{p}=0,142)$ e após $(\mathrm{p}=0,270)$ a aula, o que evidenciou que ambos os grupos incrementaram seu conhecimento após a aula teórica.

Tabela 1 - Resultados dos sujeitos $(n=31)$ referentes às questões da avaliação teórica aplicada antes e após a aula expositivo-dialogada. Ribeirão Preto-SP, 2009

\begin{tabular}{lcc}
\hline & Antes da aula expositivo-dialogada & Após a aula expositivo-dialogada \\
\hline Grupos & Média $(\%) \pm \mathbf{d p}^{*}$ & Média $(\%) \pm \mathbf{d p}^{*}$ \\
\hline Grupo 1 intervenção $(\mathrm{n}=15)$ & $32 \pm 13$ & $78 \pm 11$ \\
Grupo 2 controle $(\mathrm{n}=16)$ & $39 \pm 12$ & $82 \pm 7$ \\
Grupo 1 e Grupo 2 $(\mathrm{n}=31)$ & $36 \pm 13$ & $80 \pm 9$ \\
Valor de $\mathrm{p}^{\ddagger}$ & 0,142 & 0,270 \\
$95 \% \mathrm{IC}^{+}$ & $-0,164 ; 0,248$ & $-0,108 ; 0,316$ \\
\hline
\end{tabular}

* desvio-padrão; ${ }^{\dagger}$ 95\% intervalo de confiança; ${ }^{\ddagger}$ teste $\mathrm{t}$ de Student.

Em relação ao desempenho na avaliação prática dos olhos a média percentual de acertos foi de $76 \pm 7$ no grupo que utilizou o simulador (Grupo 1) e de $21 \pm 11$, no grupo controle $(p<0,001)$.

Tabela 2 - Resultados dos sujeitos $(n=31)$ relacionados à avaliação prática dos olhos, Ribeirão Preto-SP, 2009

\begin{tabular}{lc}
\hline Grupos & $\begin{array}{c}\text { Avaliação prática } \\
\text { dos olhos } \\
\text { Média (\%) } \pm \text { dp* }\end{array}$ \\
\hline Grupo 1 intervenção $(n=15)$ & $76 \pm 7$ \\
Grupo 2 controle $(n=16)$ & $21 \pm 11$ \\
Diferença & $-0,033$ \\
Valor de $p^{\ddagger}$ & 0,000 \\
$95 \%$ IC ${ }^{\dagger}$ & $-0,336 ;-0,478$
\end{tabular}

* desvio-padrão; † 95\% intervalo de confiança; ${ }^{\ddagger}$ teste $\mathrm{t}$ de Student

\section{DISCUSSÃO}

Os dados deste estudo mostraram que o uso de simulador no ensino da avaliação clínica dos olhos influenciou no desempenho das habilidades práticas dos estudantes de enfermagem.

Considera-se, ainda, o avanço que as pesquisas com simulações têm obtido, tanto na avaliação realizada por parte dos alunos, os quais julgam esta estratégia mais realista, torna-os mais confiantes e habilidosos em uma dada competência e reduz o receio em fazê-lo diretamente nos pacientes, quanto propicia uma maior segurança aos pacientes. Logo, tal estratégia tem sido destacada como uma facilitadora do aprendizado. ${ }^{16-17}$

O ensino por simulação permite um ambiente participativo e de interatividade, utilizando cenários clínicos que replicam experiências da vida real. É óbvio que o ensino com simulação 
não busca substituir o contato entre pacientes e profissionais de saúde, pilar necessário ao desenvolvimento de habilidades desses profissionais. O ensino por simulação permite que situações previamente selecionadas, planejadas e validadas possam ser desenvolvidas até que se atinja alto nível de proficiência. Evita-se, assim, que a intervenção do profissional de saúde seja acompanhada de efeitos deletérios ou indesejáveis. Estudantes de enfermagem têm demonstrado dificuldades no aprendizado das técnicas, na receptividade do paciente e no controlar dos sentimentos de insegurança, medo e timidez. ${ }^{18}$

A utilização dos simuladores permite a apresentação de diversos cenários, inclusive os considerados raros, dentro de um ambiente de controle. Outro aspecto relevante da simulação é que o aprendizado realizado na situação na qual o conhecimento é requerido é muito mais eficaz, permitindo a evocação dos conhecimentos e das habilidades necessárias de forma mais rápida e eficiente. ${ }^{18}$

Contudo, o desenvolvimento de habilidades para a obtenção dos dados por meio do exame físico e sua interpretação tem se tornado um desafio nas escolas de enfermagem. Cada vez mais, exige do aluno a integração dos conhecimentos obtidos nas disciplinas de anatomia, fisiologia, patologia e semiologia. Sem tal conhecimento, o enfermeiro enfrentará dificuldades em identificar os diagnósticos de enfermagem, propor um plano de cuidados, implementar e avaliar este plano, uma vez que são etapas inter-relacionadas e interdependentes. ${ }^{19}$

Destaca-se, também, que a perícia no exame é desenvolvida com a sua prática, bem como a interpretação do que é visto e ouvido. Considerando que apesar da média de acertos em nossos achados, na avaliação cognitiva, após a aula expositivo-dialogada ser de $80 \%$, o grupo que não utilizou o simulador demonstrou o seu desempenho em acertos na avaliação prática de aproximadamente $20 \%$. Por outro lado, o grupo que utilizou o simulador acertou em seu desempenho 3,6 vezes mais na avaliação prática do que o grupo que não utilizou o simulador. Neste sentido, o uso de simuladores no ensino de semiologia pode favorecer o desenvolvimento de habilidades necessárias para a prática subsequente. ${ }^{6}$

$\mathrm{Na}$ enfermagem, especificamente no ensino-aprendizagem da assistência, faz-se necessária a atuação conjunta de professor e aluno, na busca da definição e efetivação de novas formas de ensinar, para que possa se concretizar a ação de apreendê-lo. ${ }^{20}$
Desta maneira, no ensino de enfermagem, deve-se integrar o conteúdo de semiologia nas atividades práticas das demais disciplinas, em níveis crescentes de complexidade durante a formação profissional. Nessa direção, nota-se constantes discussões de propostas pedagógicas na implementação e avaliação de mudanças curriculares nos cursos de graduação em enfermagem.

Com o uso da simulação, na avaliação dos alunos, há a possibilidade de analisar o desenvolvimento de habilidades procedimentais e de pensamento crítico, bem como a construção de conhecimentos específicos, refletindo na melhor qualidade de cuidado ao paciente. ${ }^{5}$

O grande desafio na formação do enfermeiro é transpor o que é determinado pelas Novas Diretrizes Curriculares, ao formar profissionais que superem o domínio teórico-prático exigido pelo mercado de trabalho, enquanto agentes inovadores e transformadores da realidade, inseridos e valorizados no mundo do trabalho.

Em relação à avaliação clínica do olho, uma transformação da realidade seria o uso do oftalmoscópio pelo enfermeiro. Sabemos que o seu uso permite identificar alterações oculares, como também detectar doenças sistêmicas que afetam o olho, com destaque à Retinopatia Diabética (RD). A RD é a causa mais frequente de cegueira não infecciosa, e um problema de saúde pública mundial. A prevalência da RD após 15 anos de diabetes varia de $97 \%$ para a DM tipo 1 e $80 \%$ nos diabéticos tipo 2. O diagnóstico precoce da RD é realizada por meio de um simples exame de fundo de olho, com o uso do oftalmoscópio. Esse diagnóstico pode evitar, com medidas terapêuticas sistêmicas e oculares, a progressão da referida doença para formas mais graves, prevenindo, desta forma, a redução da visão. ${ }^{21}$

Nessa direção, estimular a avaliação clínica dos olhos por meio do uso de simuladores torna-se uma possibilidade de construção do conhecimento e das habilidades práticas em enfermagem. Ao se refletir sobre o processo de formação do enfermeiro na contemporaneidade, há que se considerar a resistência às mudanças, a pouca reflexão sobre a docência, o distanciamento dos serviços de saúde, com o reforço à clássica dicotomia entre o pensar e o fazer.

Algumas escolas vêm buscando estratégias para superação desses desafios, tais como a discussão em oficinas pedagógicas sobre a formação crítica e reflexiva e o conceito de competências, cada vez mais investindo na formação de co- 
missões permanentes de avaliação, capacitação permanente de docentes e inserção dos alunos em núcleos de pesquisa e orientação acadêmica, como também, a busca incessante de metodologias que articulem teoria prática, dentre outras. ${ }^{22}$

Essas são algumas das contradições que necessitam de enfrentamento e superação para melhoria da formação do enfermeiro. Portanto, aponta-se para os educadores, que o ensino de avaliação clínica dos olhos em enfermagem, com o uso de simuladores, é uma possibilidade no enfrentamento dos desafios, que pode desencadear as transformações necessárias na formação dos enfermeiros e, consequentemente, na prática assistencial.

\section{CONCLUSÕES}

Ao avaliar o uso de simuladores no ensino da avaliação clínica dos olhos no Curso de Graduação em Enfermagem de uma escola do interior paulista, foi encontrado alto percentual de acertos na avaliação teórica, após a aula expositivo-dialogada sobre a avaliação clínica dos olhos, $80 \%( \pm 9)$, que reflete o bom aproveitamento da atividade teórica ministrada.

Em relação à avaliação prática clínica dos olhos houve diferença nítida no desempenho dos dois grupos. A média percentual de acertos do Grupo 1, que utilizou o simulador, foi substancialmente maior (76 \pm 7 ), quando comparada ao Grupo 2, que teve somente o ensino tradicional e não utilizou o simulador (21 \pm 11$)$, com significância estatística $(p<0,01)$. O uso dos simuladores na avaliação clinica dos olhos possibilitou aumentar os conhecimentos teóricos e as habilidades práticas dos estudantes.

Contudo, concorda-se que o desenvolvimento de habilidades para a obtenção dos dados por meio do exame físico e sua interpretação tem se tornado um desafio nas escolas de enfermagem, uma vez que exige do aluno a integração dos conhecimentos das disciplinas de anatomia, fisiologia, patologia e semiologia. Dessa forma, avanços em simulação são de extrema importância em enfermagem.

Destaca-se como limitação do presente estudo, a utilização de simulador de baixa fidelidade, e aponta-se como imperativo, a realização de estudos futuros, utilizando-se simuladores de moderada a alta fidelidade, para o avanço no ensino de enfermagem.

\section{REFERÊNCIAS}

1. Carvalho EC. Desafios no desenvolvimento de competências de enfermeiros. Rev Latino-am Enfermagem. 2008 Set-Out; 16(5):799-800.

2. Overstreet $\mathrm{M}$. The use of simulation technology in the education of nursing students. Nurs Clin North Am. 2008 Dez; 43(4):593-603.

3. Dillard N, Sideras S, Ryan M, Carlton KH, Lasater $\mathrm{K}$, Siktberg L. A collaborative project to apply and evaluate the clinical judgment model through simulation. Nurs Educ Perspect. 2009 Mar-Abr; 30(2):99-104.

4. Peteani LA. Enhancing clinical practice and education with high-fidelity human patient simulators. Nurse Educ. 2004 Jan-Fev; 29(1):25-30.

5. Jeffries PR, McNelis AM., Wheeler CA. Simulation as a vehicle for enhancing collaborative practice models. Crit Care Nurs Clin North Am. 2008 Dec; 20(4):471-80

6. Silva CMC, Sabóia VM, Teixeira ER. O ensino do exame físico em suas dimensões técnicas e subjetivas. Texto Contexto Enferm. 2009 Jul-Set; 18(3):458-65.

7. Baranauskas MB, Margarido CB, Panossian C, Silva ED, Campanella MA, Kimachi PP. Simulação de bloqueios periféricos guiados por ultra-som: curva de aprendizado dos residentes de anestesiologia do CET-SMA/HSL. Rev Bras Anestesiol. 2008 Mar-Abr; 58(2):106-11.

8. Cicarelli DD, Coelho RB, Benseñor FEM, Vieira JE. Importância do treinamento de residentes em eventos adversos durante anestesia: experiência com o uso do simulador computadorizado. Rev Bras Anestesiol. 2005 Mar-Abr; 55(2):151-7.

9. Cogo ALP, Pedro ENR, Silva APSS, Schatkoski AM, Catalan VM, Alves RHK. Objetos educacionais digitais em enfermagem: avaliação por docentes de um curso de graduação. Rev Esc Enferm USP. 2009 Jun; 43(2):295-9.

10. Dal Sasso GTM, Souza ML. A simulação assistida por computador: a convergência no processo de educar-cuidar da enfermagem. Texto Contexto Enferm. 2006 Abr-Jun; 15(2):231-9.

11. Melo FNP, Damasceno MMC. A construção de um software educativo sobre ausculta dos sons respiratórios. Rev Esc Enferm USP. 2006 Dez; 40(4):563-5.

12. Barbosa SFF, Marin HF. Simulação baseada na web: uma ferramenta para o ensino de enfermagem em terapia intensiva. Rev Latino-am Enfermagem [online]. 2009 [acesso 2010 Jun 02]; 17(1). Disponível em: http://www.scielo.br/pdf/rlae/v17n1/pt_02. pdf

13. Seropian MA, Brown K, Gavilanes JS, Driggers B. Simulation: not just a manikin. J Nurs Educ. 2004 Abr; 43(4):164-9. 
14. Lúcio IML, Cardoso MVLML, Almeida PC. Investigação do reflexo vermelho em recém-nascidos e sua relação com fatores da história neonatal. Rev Esc Enferm USP. 2007 Jul; 41(2):222-8.

15. Goyatá SLT. Diagnósticos de Enfermagem de pacientes adultos que sofreram queimaduras e de seus familiares no período próximo à alta hospitalar [tese]. Ribeirão Preto (SP): Universidade de São Paulo. Escola de Enfermagem de Ribeirão Preto; 2005.

16. Reilly A, SprattS. The perceptions of under graduate student nurses of high-fidelity simulation based learning: A case report from the University of Tasmania. Nurse Educ Today. 2007 Aug; 27(6):542-50.

17. Barsuk JH, Mcgaghie WC, Cohen ER, O'leary KJ, Wayne DB. Simulation-based mastery learning reduces complications during central venous catheter insertion in a medical intensive care unit. Crit Care Med. 2009 Oct; 37(10):2697-701.

18. Mizoi CS, Kaneko RMU, Filho CAM. A simulação realística como estratégia de treinamento para profissionais da saúde. Einstein: Educ Contin Saúde. 2007; 5(3):100-1.
19. Souza MF, Barros ALBL, Michel JL Marlene, Nobrega MML, Ganzarolli MZ. Bases teórico-metodológicas para a coleta de dados de enfermagem. In: Barros ALBL, organizador. Anamnese e exame físico: avaliação diagnóstica de enfermagem no adulto. Porto Alegre (RS): Artmed; 2010. p.21-54.

20. Anastasiou LGC, Alves LP. Processos de ensinagem na universidade: pressupostos para as estratégias de trabalho em aula. $6^{\text {a }}$ ed. Joinville (SC): Ed. Univille; 2006.

21. Sociedade Brasileira de Diabetes. Tratamento e acompanhamento do diabetes mellitus: diretrizes da Sociedade Brasileira de Diabetes. Rio de Janeiro (RJ): Diagraphic; 2009.

22. Silva MG, Fernandes JD, Teixeira GAS, Silva RMO. Processo de formação da(o) enfermeira(o) na contemporaneidade: desafios e perspectivas. Texto Contexto Enferm [online]. 2010 Jan-Mar [citado 2011 Abr 11]; 19(1):176-84. Disponível em: http:/ /www. scielo.br $/$ scielo.php?script=sci_arttext\&pid=S010407072010000100021\&lng=pt 